STROMA

\title{
Environmental issues
}

It has become evident that the tissue surrounding a tumour can have as important a role in cancer development as the genetic changes that occur in the tumour cells themselves. How does the microenvironment mediate these carcinogenic effects? Derek Radisky et al. report in Nature that, in mammary tissue, overproduction of matrix metalloproteinase-3 (MMP3) causes surrounding cells to increase production of reactive oxygen species (ROS), leading to DNA damage and genomic instability, and eventually to malignant transformation.

MMPs are extracellular-matrixdegrading enzymes that are produced by the tumour stroma and have wellknown roles in late-stage tumour progression, promoting cell recruitment, and invasion and metastasis. Radisky et al. have been trying to determine how some MMPs, such as MMP3, can also directly transform mammary epithelial cells.

Changes in the morphology of MMP3-transformed mammary epithelial cells indicated that alterations in a cytoskeletal regulatory protein might be involved. In examining expression levels of a number of cytoskeletal regulators, the authors found that an alternatively spliced form of the GTPase RAC1 (RAC1b) was overproduced in MMP3-transformed cells. Interestingly, this isoform had been previously reported to be upregulated in human breastand colorectal-tumours, and to have transforming capabilities in cultured cells.

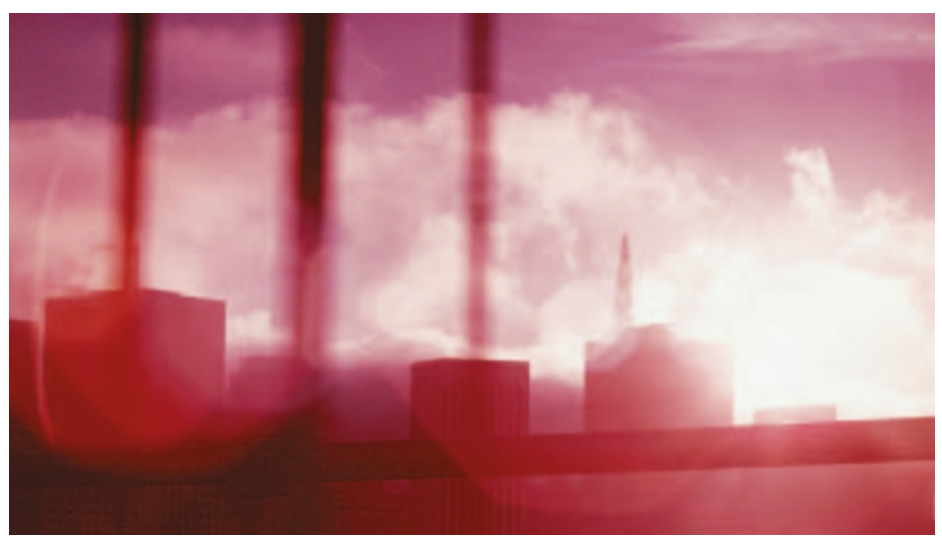

How could RAC1b mediate transformation? In addition to its roles in regulating formation of cytoskeletal structures, RAC1 can also stimulate production and release of mitochondrial superoxide into the cytoplasm. The authors showed that treatment of cells with MMP3 produced RAC1-dependent increases in cellular levels of ROS, which led to various cellular changes that are associated with tumorigenesis: altered expression of transcription factors, induction of the epithelial to mesenchymal transition, along with increased cell motility and invasiveness. All these changes were prevented with the ROS-quenching agent $N$-acetyl cysteine (NAC). Most importantly, MMP3-induced ROS production also led to DNA damage, as well as genomic amplifications and deletions in cells - hallmarks of tumorigenesis.

It is still not clear how increased extracellular levels of MMP3 upregulate this alternative splice product of RAC1, although it could be through the ability of MMP3 to cleave the extracellular receptor protein E-cadherin (CDH1), which initiates an intracellular signalling cascade. The search is now on for other microenvironmental factors that can also stimulate phenotypic and genotypic transformation of normal cells.

Kristine Novak

(1) References and links ORIGINAL RESEARCH PAPER Radisky, D.C. et al. RAC1b and reactive oxygen species mediate MMP-3-induced EMT and genomic instability. Nature 436, 123-127 (2005)

\section{TRIAL WATCH}

\section{Inflammatory trends}

A study of incidence and survival trends for inflammatory breast carcinoma (IBC) shows that IBC incidence rates increased throughout the 1990s, and that substantial racial differences in incidence rates are evident.

Paul Levine and colleagues analysed Surveillance, Epidemiology and End Results (SEER) Program data for breast cancer cases diagnosed between 1988 and 2000. Estimates of IBC incidence have varied widely, possibly due to differing classification criteria for IBC, which might have included cases of non-inflammatory, locally-advanced breast cancer (LABC). This analysis used a comprehensive clinical and pathological definition of IBC, using SEER codes for tumour size and tumour extension, as well as morphology (that is, embolization of the dermal lymphatic vessels). Of the 180,224 cases included, 3,648 were classified as IBC, 3,636 as LABC and the remainder as breast cancer confined to the breast tissue and fat (classified as T1-T3 by the American Joint Committee on Cancer staging).

Incidence rates of IBC increased from 2.0 in 1998-1990 to 2.5 in 1997-1999 - an increase of 25\%. Over the same time, incidence of LABC and T1-T3 breast cancers decreased, which might be associated with early detection owing to mammography screening. By contrast, IBC is difficult to detect by mammography because it is a diffuse tumour obscured by increased breast density. IBC incidence rates were higher in black women (3.1) than in white women (2.2). Women diagnosed with IBC had poorer median survival ( 2.9 years) than women diagnosed with LABC ( 6.4 years) or T1-T3 breast cancer ( $>10$ years). Black women with IBC or LABC had poorer survival than white women. Several groups are working to define the molecular profile of IBC, which might help to determine why IBC incidence is rising and why it is such an aggressive, therapy-resistant disease.

ORIGINAL REFERENCE Hance, K. W. et al. Trends in inflammatory breast carcinoma incidence and survival: the Surveillance, Epidemiology and End Results Program at the National Cancer Institute J. Natl Cancer Inst. 97, 966-975 (2005)

\section{Two-pronged attack}

ZD6474 is an orally bioavailable inhibitor of both vascular endothelial growth factor receptor-2 (VEGFR2) tyrosine-kinase and epidermal growth factor receptor (EGFR) tyrosine-kinase activity. At the 11th World Conference on Lung Cancer in July 2005, promising results were presented from two phase II trials with ZD6474 in the second-line treatment of non-small-cell lung cancer (NSCLC).

Trial 003 was led by Ronald Natale (Cedars-Sinai Outpatient Cancer Center, Los Angeles, USA) and compared ZD6474 and gefitinib as monotherapies in patients with advanced NSCLC. Patients receiving ZD6474 had a higher objective response rate and longer progression-free survival (PFS) than those on gefitinib.

Trial 006, a combination-therapy study, showed that patients receiving ZD6474 plus docetaxel had an increased median PFS compared with those receiving docetaxel alone. The lead investigators on this study were John Heymach (Dana-Farber Cancer institute, Boston, USA) and Roy Herbst (University of Texas MD Anderson Cancer Center, Texas, USA).

Recruitment for phase III trials of ZD6474 will start later this year. WEBSITE http://www.2005worldlungcancer.com/2005WLC/ 